\title{
Progresses of the Dark Matter Particle Explorer experiment
}

\author{
Qiang Yuan*; on behalf of the DAMPE collaboration \\ ${ }^{1}$ Key Laboratory of Dark Matter and Space Astronomy, Purple Mountain Observatory, Chinese \\ Academy of Sciences, Nanjing 210008, China \\ ${ }^{2}$ School of Astronomy and Space Science, University of Science and Technology of China, Hefei \\ 230026, China \\ E-mail: yuanq@pmo.ac.cn
}

The Dark Matter Particle Explorer (DAMPE) is a satellite-borne, calorimetric type, high-energyresolution space cosmic ray and gamma-ray detector. It operates stably in space for more than three years since its launch in December, 2015. Precise measurements of the total electron plus positron spectrum and the proton spectrum in wide energy ranges have been obtained, which are expected to shed new light on our understanding of the cosmic ray origin and the nature of dark matter particles. The current status of the mission and its latest physical results will be presented.

36th International Cosmic Ray Conference -ICRC2019-

July 24th - August 1st, 2019

Madison, WI, U.S.A.

\footnotetext{
* Speaker.

$\dagger$ The DAMPE mission is funded by the strategic priority science and technology projects in space science of Chinese Academy of Sciences. In China the data analysis is supported in part by the National Key Research and Development Program of China (No. 2016YFA0400200), the National Natural Science Foundation of China (Nos. 11525313, 11622327, 11722328, U1738205, U1738207, U1738208, 11851305), the strategic priority science and technology projects of Chinese Academy of Sciences (No. XDA15051100), the 100 Talents Program of Chinese Academy of Sciences, and the Young Elite Scientists Sponsorship Program. In Europe the activities and the data analysis are supported by the Swiss National Science Foundation (SNSF), Switzerland; the National Institute for Nuclear Physics (INFN), Italy.

${ }^{\ddagger}$ For collaboration list see PoS (ICRC2019) 1177.
} 


\section{Introduction}

The Dark Matter Particle Explorer (DAMPE) is a satellite-borne, calorimetric type, highenergy particle detector dedicated to the indirect detection of dark matter (DM) in space and astrophysical studies $[1,2]$. The DAMPE satellite was launched to a $500 \mathrm{~km}$ Sun-synchronous orbit on December 17th, 2015. It operates very smoothly in orbit since then. The trigger rate of the DAMPE detector is about $60 \mathrm{~Hz}$, and the total number of events exceeds 6.3 billion as of June 30th, 2019.

The DAMPE detector consists of four sub-detectors. They are the plastic scintillator detector (PSD; [3]), the silicon-tungsten tracker converter (STK; [4]), the BGO imaging calorimeter [5], and the neutron detector (NUD; [6]). The PSD measures the (absolute) charge of incident particles via the ionization effect, and is used as veto of charged CRs for $\gamma$-ray detection. The STK measures the trajectory and also charge of particles. The BGO is to measure the energy of incident particles and provide electron-proton discrimination based on shower morphologies. The NUD provides additional electron-proton discrimination through the neutron content difference between hadronic and electromagnetic showers. The DAMPE detector is optimized for observations of CR electrons (including positrons; hereafter CREs) and $\gamma$-rays up to $10 \mathrm{TeV}$ energies with unprecedentedly high energy resolution and electron-proton discrimination capability, thanks to the design of a full-absoption, thick ( $\sim 32$ radiation lengths) calorimeter. It can thus improve significantly the measurements of the CRE spectrum and the sensitivity of searching for DM particles. The on-orbit calibration shows that each sub-detector works very stably since the lanuch $[7,8]$.

\section{On-orbit calibration and performance}

DAMPE enables two modes for data acquisition (DAQ), the "dedicated mode" for the calibration of the electronics linearity and pedestal, and the "continuous mode" for science data taking. Calibrations other than the electronics linearity and pedestal, including the minimum ionization particle (MIP) response, light attenuation, dynode ratios, detector alignment etc. are done with data taken in the "continuous mode". In each orbit, the DAQ system switches from the "continuous mode" to the "dedicated mode" twice - each with 40 seconds - when the satellite is at a latitude of $+20^{\circ}$. The details about the calibration of each sub-detector can be found in [7].

The PSD detector is mainly used for charge detection. The particle charge is proportional to the square root of the deposit energy in the PSD bar. For proton MIP events, the energy deposition in the PSD is about $2 \mathrm{MeV} \mathrm{cm}^{-1}$. After a series of corrections such as the attenuation of scintillation light in the PSD bar [9], the alignment of the PSD bars [10], the equalization of different bars, as well as the quenching effect, we finanly get high-resolution measurement of particle charges [11]. Figure 1 shows the charge spectrum based on two years of flight data of DAMPE [11]. Gaussian fittings show that the charge resolution is about $0.2 e$ for $\mathrm{CNO}$ and $0.3 e$ for $\mathrm{Fe}$.

The STK is primarily used to measure the direction as well as the charge (for $Z<8$ ) of particles. $\gamma$-ray photons can convert into a pair of electron and positron in the tungsten plates inserted into the STK, leaving also track imprint in the STK. The direction measurement basically uses the information of hit positions, while the charge measurement uses the signal strength along the trajectory. The calibration of the STK includes the pedestal and noise evaluation, the equalization of the VA140 chips, and the alignment of silicon sensors [12]. The spatial resolution of the STK 


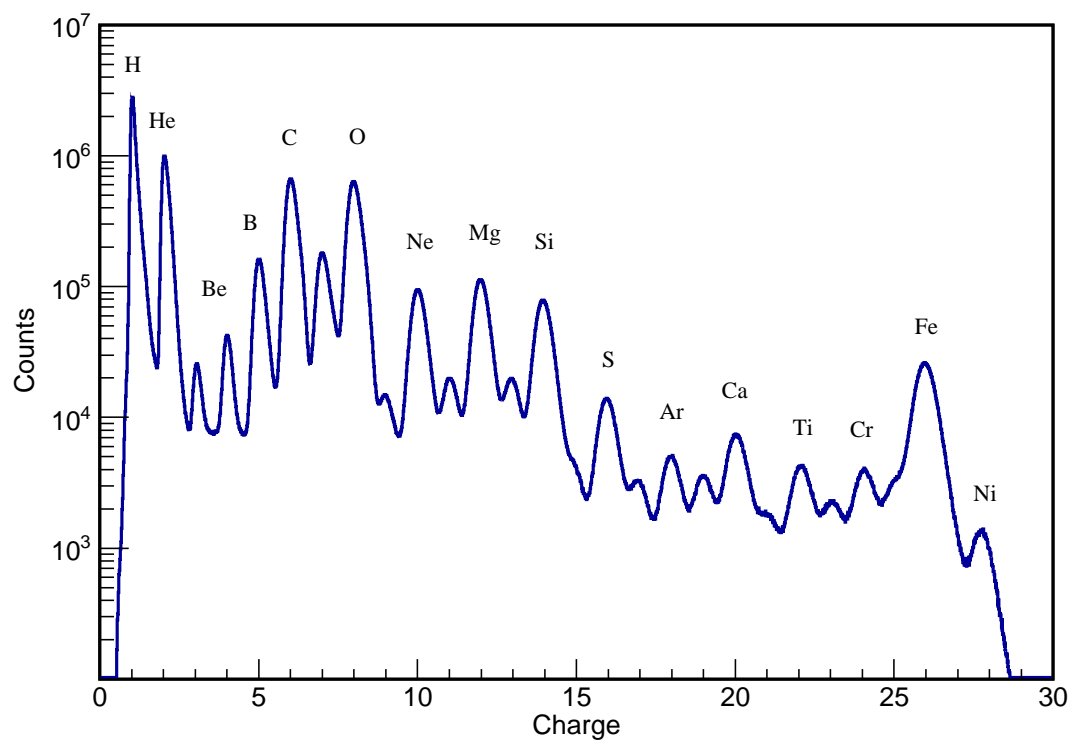

Figure 1: The charge spectrum from two years of DAMPE data. Plot from [11].

is about $50 \mu \mathrm{m}$ after the alignment procedure [12]. Monte Carlo (MC) simulations show that the angular resolution of normal incident photons is about $1.2^{\circ}\left(0.1^{\circ}\right)$ at $1(100) \mathrm{GeV}$ [2].

The BGO calorimeter is to measure the particle energy, and to discriminate CREs from protons. The shower morphology in the BGO can also be used to reconstruct the trajectory, although its resolution is limited. Actually the so-called "global track" is based on a joint assessment of the BGO track and the STK track(s), which is widely adopted in our analysis. The BGO calibration includes the pedestal calibration, the MIP response, the PMT dynode ratio evaluation, the light attenuation length calculation, and the trigger threshold determination. The beam tests verified that the energy resolution of the BGO reaches $\sim 1 \%$ for CREs when $E>100 \mathrm{GeV}$ [13].

The NUD is to measure the secondary neutrons produced by the particle shower in the BGO. The pedestals of NUD are calibrated using periodic trigger events during the "dedicated mode" every day. The gate open for neutron signal integration is set to be $2.5 \mu$ s to suppress the charged secondary particles.

The satellite travels through the South Atlantic Anomaly (SAA) region nearly 7 times per day. The very intensive particle hit rate within the SAA region may induce severe pileup effects, which hinders science analyses. The trigger rates as stored in the house keeping data are used to determine the boundary of the SAA region. The live time is computed through subtracting the time when the satellite is in the SAA region, the instrumental dead-time, and the on-orbit calibration time. Photon events from bright $\gamma$-ray sources are used to correct the boresight alignment [7, 14].

\section{Measurements of the CRE spectrum}

High-energy CREs are ideal probe of nearby CR sources due to their limited propagation distance. They are also sensitive to search for the annihilation or decay of DM particles, due 
primarily to the fact that the $e^{-}$component is not much more abundant than the $e^{+}$component in CREs. The observations of CREs are challenging because of the high fluxes from the proton background. Therefore a high rejection power of $\mathrm{CR}$ protons is crucial to reliably identifying CREs. The DAMPE experiment uses shower morphologies in the BGO calorimeter to disciminate CREs from protons (heavier nuclei can be significantly rejected through charge measurement) [15]. A two-parameter method based on the quantification of the transverse and longitudinal distributions of showers is developed. The transverse spread of a shower is described by an energy-dependent root-mean-square (RMS) value of hit positions:

$$
\mathrm{RMS}_{i}=\sqrt{\frac{\sum_{j}\left(x_{j, i}-x_{c, i}\right)^{2} E_{j, i}}{\sum_{j} E_{j, i}}},
$$

where $x_{j, i}$ and $E_{j, i}$ are coordinate and deposit energy of the $j$ th bar in the $i$ th layer, and $x_{c, i}$ is the coordinate of the shower center of the $i$ th layer. The longitudinal distribution parameter uses the energy fraction of the last BGO layer ( $\mathscr{F}_{\text {last }}$ ) to describe the tail of a shower [16]. A joint parameter, $\zeta=\mathscr{F}_{\text {last }} \times\left(\sum_{i} \mathrm{RMS}_{i} / \mathrm{mm}\right)^{4} /\left(8 \times 10^{6}\right)$, is employed to select CRE candidates. We find that when setting $\zeta \leq 8.5$, a very clean CRE sample with only $\sim 2 \%$ contamination of the proton background can be obtained for $\mathrm{TeV}$ energies [16]. Figure 2 shows the scattering plot of $\sum_{i} \mathrm{RMS}_{i}$ and $\mathscr{F}_{\text {last }}$ (left panel) and the one-dimensional distribution of $\zeta$ (right panel). A clear seperation between CRE candidates and proton candidates can be seen. Independent methods using more variables can be found in [17], which gives similar results as this two-parameter method.
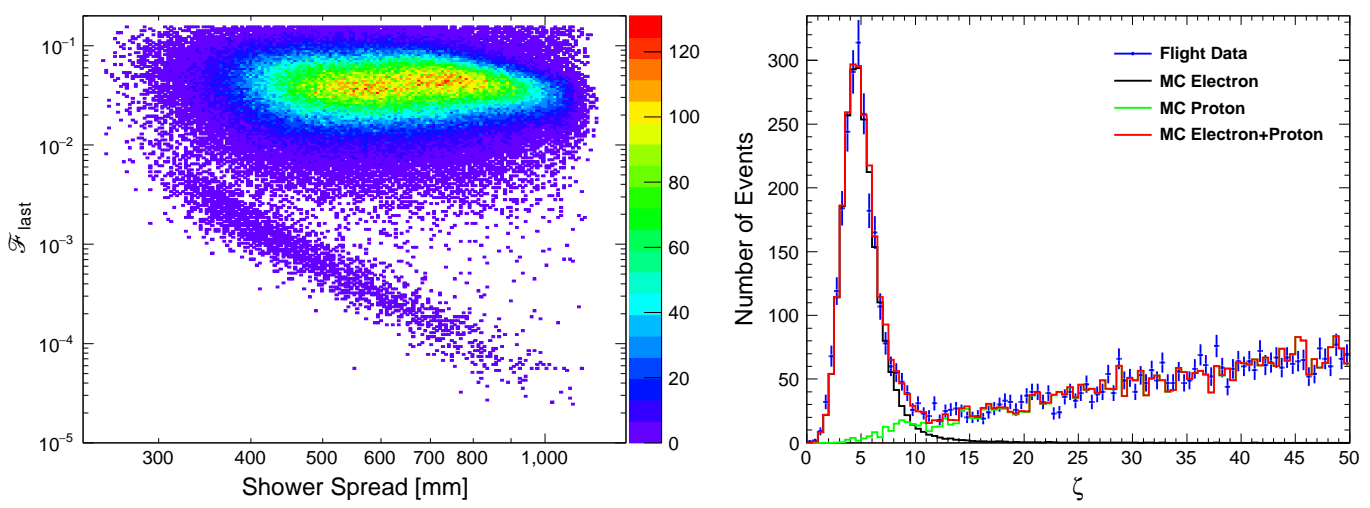

Figure 2: Left: Shower spread $\left(\sum_{i} \mathrm{RMS}_{i}\right)$ versue the last layer energy fraction $\mathscr{F}_{1}$ ast for selected events with deposited energies between $500 \mathrm{GeV}$ and $1 \mathrm{TeV}$. Right: one-dimensional distributions of the shower shape parameter $\zeta$, compared with MC simulations. Plot from [16].

Using 530 days of flight data, we measure the CRE spectrum from $25 \mathrm{GeV}$ to $4.6 \mathrm{TeV}$ with high precision [16]. Figure 3 shows the CRE spectrum measured by DAMPE, and that by AMS-02 [18], Fermi-LAT [19], CALET [20], and H.E.S.S. [21, 22]. For the first time, the DAMPE result reveals a clear spectral break of the $\mathrm{CRE}$ spectrum at $\sim 0.9 \mathrm{TeV}$, with the spectral index changing from -3.1 to -3.9 . Previously, the H.E.S.S. data together with low energy measurements by other experiments showed only weak evidence for such a break, with relatively large systematic uncertainties (shown by the shaded band in Figure 3). 


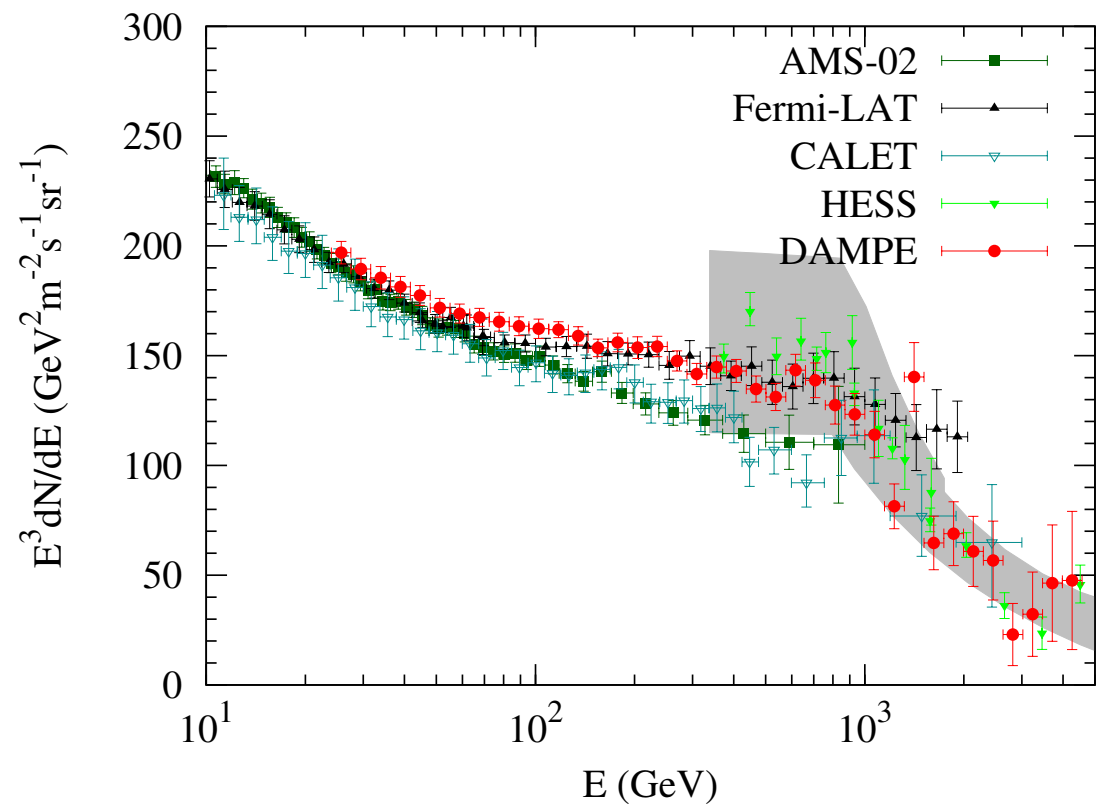

Figure 3: The CRE spectra measured by DAMPE (red dots) [16], compared with results from AMS-02 [18], Fermi-LAT [19], CALET [20], and H.E.S.S. [21, 22]. Shaded band shows the systematic uncertainties of the H.E.S.S. measurements. Plot from [24].

The DAMPE result has important implications on the understanding of the modeling of CREs. The wide energy range coverage and high precision of the DAMPE spectrum can significantly improve the constraints on the model parameters to fit the positron excess, either for astrophysical models or the DM annihilation or decay [23]. The spectral break may naturally reflect the discretness of the source distribution due to the fast cooling of TeV CREs [24]. Another interpretation ascribes the softening to the confinement and cooling of CREs during the acceleration stage [25].

\section{Measurements of the spectra of cosmic ray nuclei}

The current precise measurements of the spectra of nuclei come from the magnetic spectrometer experiments, such as PAMELA [26] and AMS-02 [27, 28]. The balloon-borne experiments such as ATIC [29] and CREAM [31, 30], and satellite experiment NUCLEON [32] extend the measurements to $100 \mathrm{TeV}$ energies, but with large statistical and/or systematic uncertainties. Interestingly, these results show hints that the energy spectra of protons, Helium, and heavier nuclei soften at $\sim 10 \mathrm{TeV}$ energies [31,32]. It is thus very important to have improved measurements with substantially higher statistics and better control of systematic uncertainties in the energy range between $\mathrm{TeV}$ and $100 \mathrm{TeV}$ to clarify the spectral behaviors of CRs. A relatively large acceptance and thick calorimeter (about 1.6 nuclear interaction lengths) of the DAMPE makes it appropriate for observations of CR nuclei.

Using 30 months of DAMPE data recorded from January 1, 2016 to June 30, 2018, we analyze the proton component in CRs. The fraction of live time is about $75.7 \%$ after subtracting the SAA passage time, the instrument dead time, and the calibration time. Events with deposited energies 
higher than $20 \mathrm{GeV}$ in the $\mathrm{BGO}$ are selected. We further requie the high-energy trigger condition of the event selection.

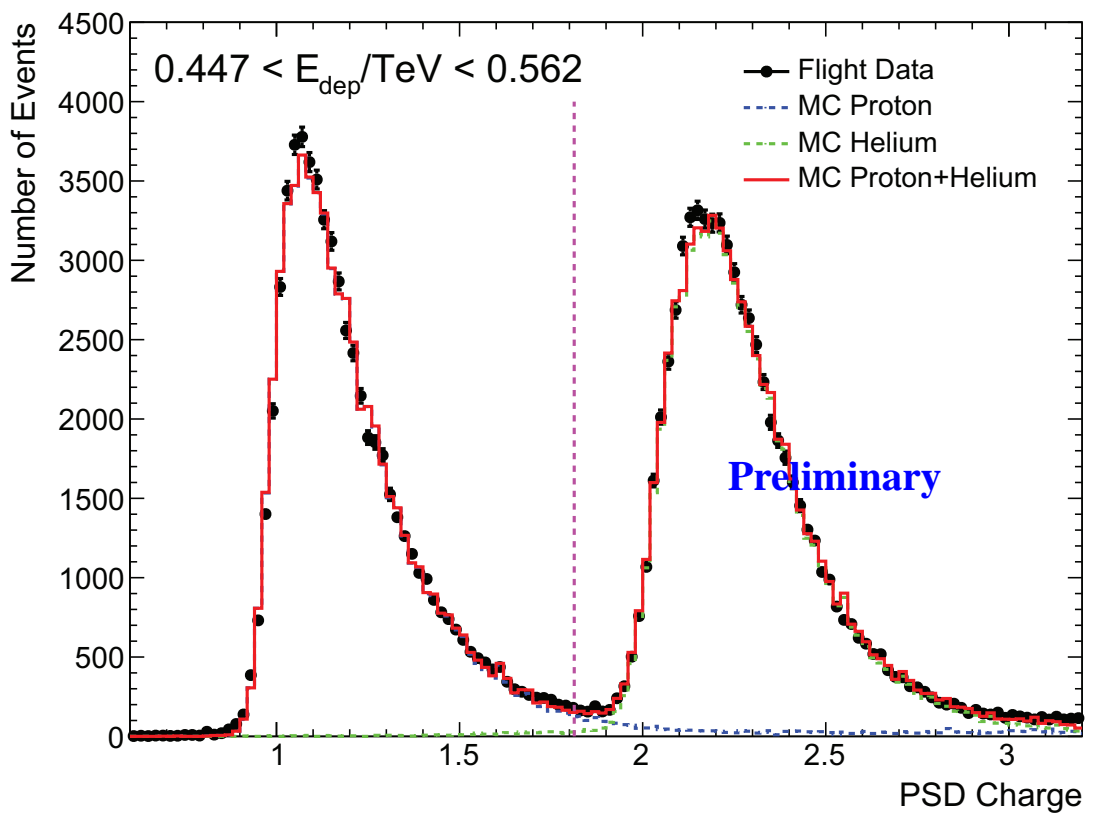

Figure 4: The PSD charge distribution of low- $Z$ nuclei of the DAMPE data, compared with simulations of protons and Helium nuclei [34].

The "PSD charge" distribution of selected events with deposited energies between 447 and $562 \mathrm{GeV}$ is shown in Figure 5. Also shown are the MC simulation results. Note that here the energy-dependent correction from the PSD signal to the particle charge has not been taken into account. For the MC simulation sample, a correction procedure has been applied to correct the mis-match with the flight data due to the imprecise modeling of the backscattering [34]. Protons are selected based on a BGO-energy-dependent cut of the "PSD charge" [34]. The Helium backgrouns is estimated according to a fitting to the charge distribution of the flight data using MC templates, which turns out to be $\lesssim 1 \%$ for deposited energies below $10 \mathrm{TeV}$ and up to $\sim 5 \%$ around 50 $\mathrm{TeV}$. The electron background is found to be extremely small thanks to the high electron-proton discrimination capability of the DAMPE [16].

We use also the MC simulations to estimate the selection efficiencies of each step, as well as the energy response matrix of proton events in the calorimeter. The proton spectrum from $40 \mathrm{GeV}$ to $100 \mathrm{TeV}$ after the spectral unfolding [35] is shown in Figure 5. The DAMPE result confirms the spectral hardenings at hundreds $\mathrm{GeV}$ revealed previously [29, 30, 26, 27, 33], and most importantly, reveals a spectral softening around $10 \mathrm{TeV}$. A smoothly broken power-law fit gives that the spectral index changes from -2.60 to -2.85 at $13.6 \mathrm{TeV}$. This softening feature may have very interesting implication in modeling the origin and propagation of CRs (e.g., [36, 37]).

Figure 6 gives the preliminary Helium spectrum from $10 \mathrm{GeV} / \mathrm{n}$ to $5 \mathrm{TeV} / \mathrm{n}$ [38]. The spectral hardening at hundreds of $\mathrm{GeV}$ energies can be seen, which is consistent with previous measurements. More detailed analysis is on-going. The analyses of the total proton plus Helium spectrum 


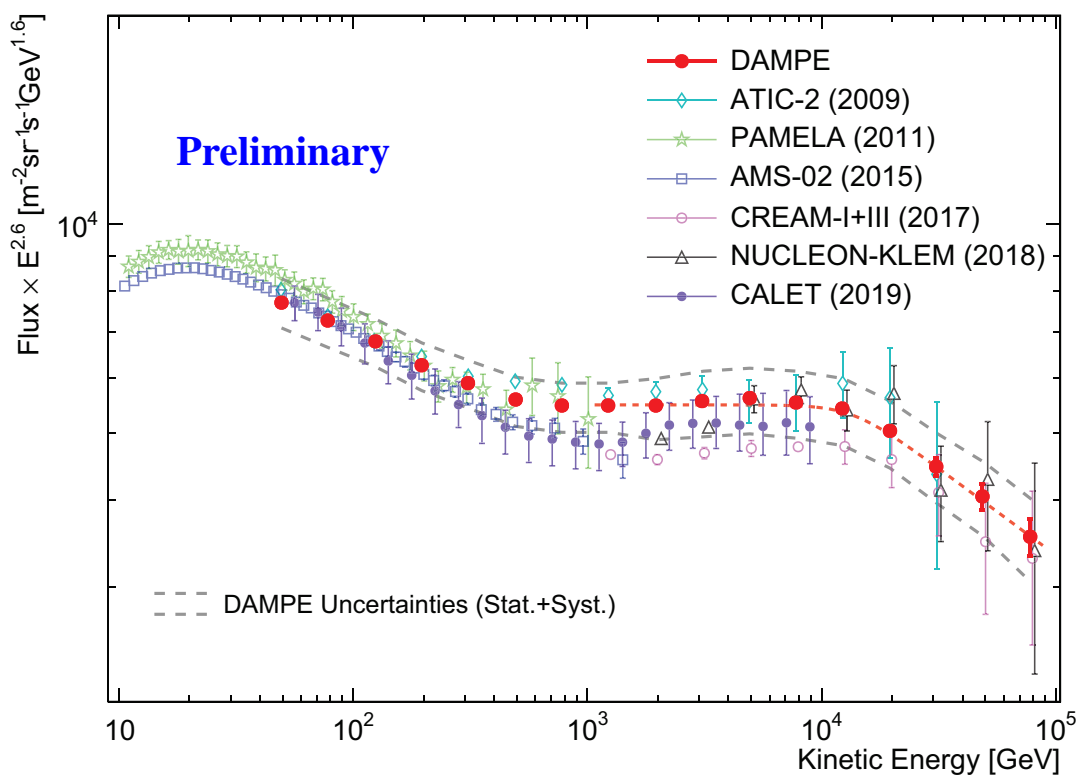

Figure 5: The proton spectrum measured by DAMPE (red dots; [34]), compared with that measured by other experiments [29, 31, 32, 26, 27, 33]. The total uncertainties including the statistical ones and the systematic ones are shown by the dashed lines.

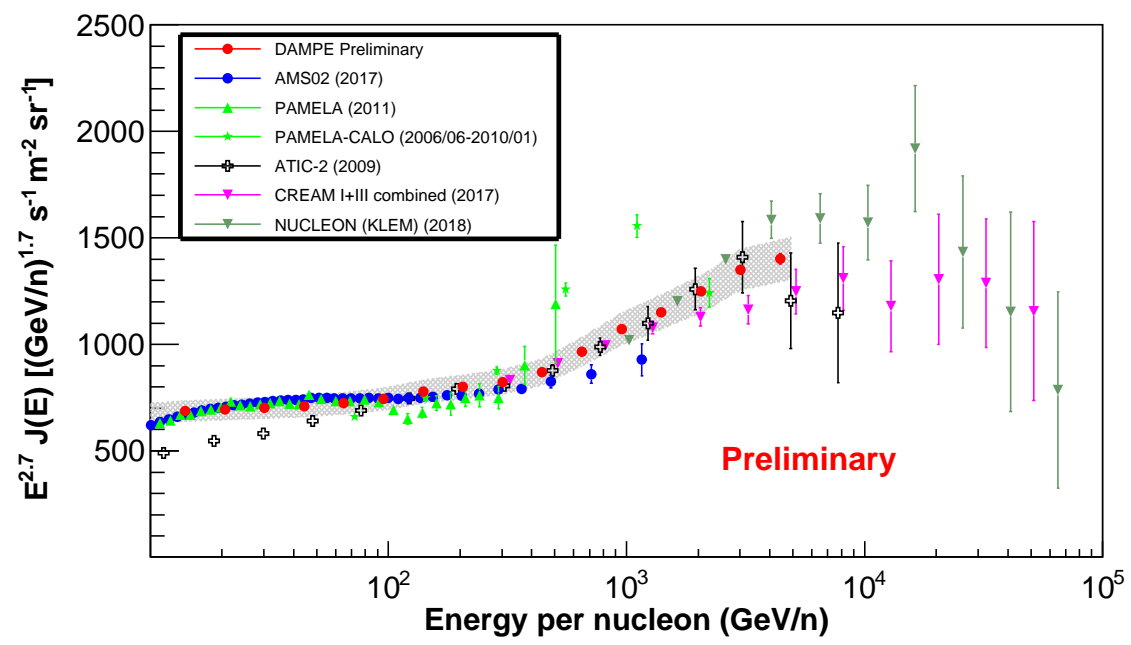

Figure 6: Preliminary result of the Helium spectrum measured by DAMPE (red dots; [38]), compared with that measured by other experiments [29, 26, 40, 31, 39, 32]. Errorbars show the statistical uncertainties and gray shaded band shows the systematic uncertainties.

[41] and heavier nuclei $[42,43]$ are also carried out, with prelimary results or analysis progresses reported in this conference. Note that dedicated simulations of the hadronic interactions beyond $100 \mathrm{TeV}$ energies for CR nuclei are necessary [44]. 


\section{Cosmic ray anisotropies}

CRs propagate diffusively in the turbulent magnetic fields of the Milky Way, which results in nearly isotropy of their arrival directions in the sky. However, tiny anisotropies were observed (e.g., [45]), reflecting important properties of the origin and propagation of CRs. Currently the detection of the anisotropies is mostly from ground or underground experiments, which have limited composition resolution and sky coverage. Furthermore, the groundbased air shower experiments also suffer from problems of calibration of the absolute efficiencies for different latitude bands. Therefore it is very important to measure the anisotropies from space direct detection experiments.

There were efforts to search for anisotropies of either CR nuclei or electrons/positrons by PAMELA [46], Fermi-LAT [47, 48], and AMS-02 [49]. No significant anisotropies were detected except that PAMELA reported a potential detection of a dipole anisotropy using its calorimeter sample of all CRs [50].

Using one year of the DAMPE data recorded in 2016, we study the anisotropies of all CRs [51]. After removing events recorded when the satellite is in the SAA region, we further select the events with maximum zenith angles of $60^{\circ}$ in the detector coordinate and deposited energies between $100 \mathrm{GeV}$ and $500 \mathrm{GeV}$ in the BGO calorimeter. Note that the primary events should have energies higher by a factor of $2 \sim 3$ in this energy range. After all these cuts, we get a total number of events of about $6.1 \times 10^{6}$. The skymap based on the selected events in the equatorial coordinate is shown in the top-left panel of Figure 7.
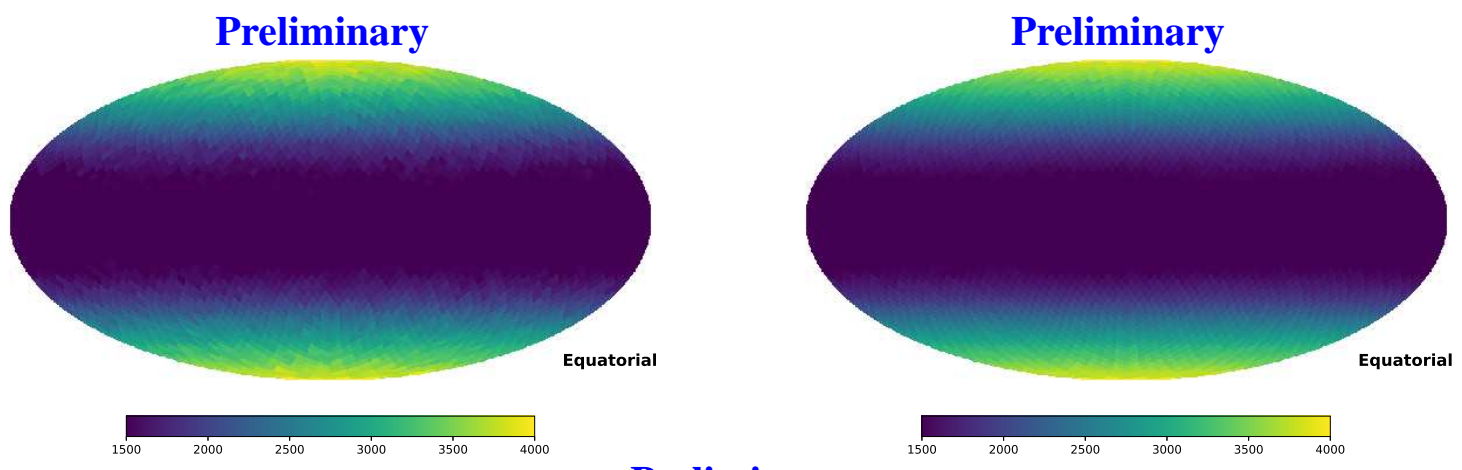

Preliminary

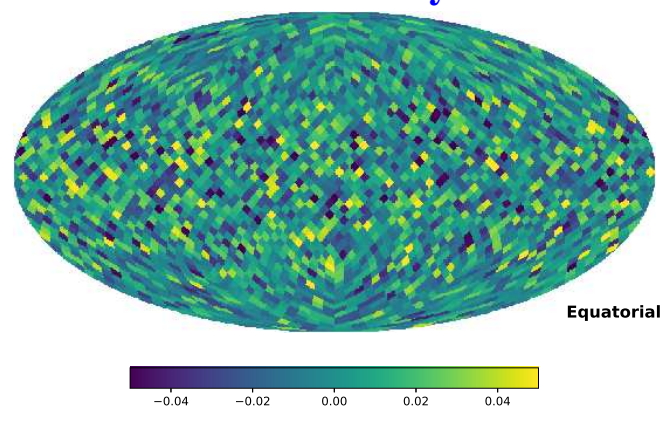

Figure 7: Skymaps of DAMPE raw events (top-left panel), the reference exposure map from the rate-based method (top-right panel), and the relative intensity map (bottom panel) [51].

The exposure of the DAMPE is highly non-uniform. To obtain a precise exposure map is crucial to the search for tiny anisotropies of CR arrival directions. The reference exposure map is 
created with the rate-based method proposed in [47]. An average of 100 reference maps is shown in the top-right panel of Figure 7. The relative intensity map, defined as $\delta I=D / R-1$, where $D$ is the data map and $R$ is the reference map, is shown in the bottom panel. The result is consistent with the null-hypothesis. The $95 \%$ confidence level upper limit of the dipole amplitude is $6.7 \times 10^{-3}$ for one year data sample. Further improvement of the sensitivity of anisotropy searches can be expected with more data added.

\section{Gamma-ray astronomy}

Gamma-ray astronomy is one of the main scientific objectives of the DAMPE [52]. Thanks to its high energy resolution, the DAMPE is expected to play a key role in searching for monochromatic $\gamma$-ray line emission. The observations of $\gamma$-ray transients are also very interesting and important in the multi-messenger era, particularly in the case that gravitational waves [53] and veryhigh-energy astrophysical neutrinos [54] have been discovered.

Compared with charged CRs, the flux of $\gamma$-rays is significantly smaller by a factor of $10^{5} \sim 10^{6}$. An effect way to pick out $\gamma$-rays with little contamination of charged CRs is the key for studies of the $\gamma$-ray astronomy. A dedicated algorithm has been developed to resolve $\gamma$-rays from the flight data [55]. It includes joint efforts of electromagnetic and hadronic shower discrmination with the BGO calorimeter and the veto of charged particles with the PSD detector. With such a method, we can reach an effective acceptance of $\sim 0.19(0.11) \mathrm{m}^{2} \mathrm{sr}$ at $10\left(10^{3}\right) \mathrm{GeV}$ of $\gamma$-ray detection, with a purity of $\sim 99 \%$ for energies higher than $5 \mathrm{GeV}$ [55].

Using three years of the DAMPE data, we have searched for possible line-like emission between $10 \mathrm{GeV}$ and $300 \mathrm{GeV}$ for different sky regions around the Galactic center and the directions of a class of nearby galaxy clusters [56]. The scientific tool of DmpST and the embedded instrumental response functions (IRFs) based on MC simulations have been used [57]. The energy resolution of the selected sample, which is not optimized for line searches yet, is about $(1.5 \sim 2) \%$. The left panel of Figure 8 shows the spectral energy distribution of $\gamma$-rays in a region with $90^{\circ}$ radius around the Galactic center excluding the outer Galactic plane with $|b|<5^{\circ}$ and $|l|>6^{\circ}$ (defined as R90 region in [58]). Using a sliding window technique, we search for possible line-like signal in the data with an unbinned maximum likelihood method. No significant line-like emission has been found, and we thus derive the $95 \%$ confidence level upper limits as shown in the right panel of Figure 8. The DAMPE observations give comparable sensitivities for line emission searches compared with the 5.8 year results of Fermi-LAT [59], illustrating the advantages of high energy resolution. Optimized analysis with better resolution of the data sample is expected to further improve the sensitivities.

Pulsars are a class of highly magnetized, rotating neutron stars which are of particular interests of physics in strong magnetic field and high density of matter. Quite a number of pulsars are very bright $\gamma$-ray sources in the sky [60]. A sample of nearly 20 pulsars are identified with pulsations using the DAMPE data. The light curves and spectra of some of them are presented in [61].

\section{Solar modulation and solar activities}

Low energy CRs are affected by the magnetic fields carried by the outgoing solar winds, 

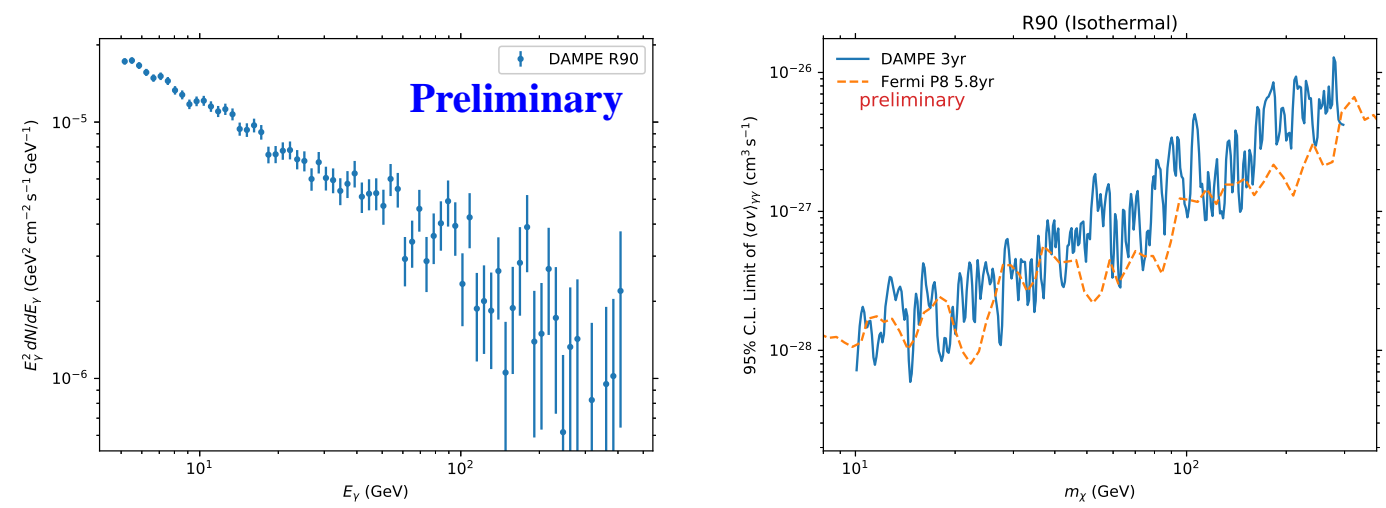

Figure 8: Left: fluxes of $\gamma$-rays in the R90 region. Right: the 95\% confidence level upper limits of the DM annihilation cross sections assuming an isothermal profile of the DM density. Plots from [56].

known as the solar modulation. The explosive activities of the Sun also generate various kinds of effects on the fluxes of CRs such as the Forbush decrease, the global merged interaction regions, and the solar energtic particles. The time evolution of CRs is thus a unique probe of the heliosphere environment and solar activities. Studies of the time evolution or solar-activity-related CR flux changes by AMS-02 [62, 63] and PAMELA [64, 65, 66] showed interesting properties of the particle transport in the solar system. Compared with PAMELA and AMS-02, the DAMPE may have unique advantages in studying the fine time and spectral structures of the CRE fluxes, due to the facts that, a high inclination angle $\left(\sim 97^{\circ}\right)$ enables reaches of the polar region which is less affected by the geomagnetic rigidity cutoff, and a relatively large acceptance $\left(\sim 0.35 \mathrm{~m}^{2} \mathrm{sr}\right)$ enables enough statistics to reveal very short-term variabilities of the fluxes.

We measure the monthly CRE fluxes from April, 2016 to June, 2019, from $2 \mathrm{GeV}$ to $20 \mathrm{GeV}$ [67]. The flux ratios relative to that of April, 2016, for a few energy bins are shown in Figure 9. A clear anti-correlation between the CRE fluxes and the sunspot numbers [68] can be seen, as expected for the solar modulation effect. For energies higher than $\sim 10 \mathrm{GeV}$, the CRE fluxes become less affected by the solar modulation. A time delay between the solar activities and the impacts on the CRE fluxes $[63,69]$ is not clearly seen because of the relatively short time coverage of the current analysis.

On September 7, 2017, a big solar flare occured. We study the impact of such a flare on the low energy CRE fluxes in detail. Figure 10 shows the relative time profile of the CRE fluxes for 6 hour binning with respect to the average flux of August, 2017. Clear Forbush decreases can be seen after the solar flare. After reaching the minima, the CRE fluxes recover to the normal values for a time scale of about one week, similar with that found by PAMELA [64]. Both the amplitudes and recovery time decrease with energy (see [67] for more details). Such behaviors are consistent with one class of solar disturbances causing the Forbush decrease, which is probably associated with a complicated shock-associated interplanetary coronal mass ejection disturbance with a large radial extent [70]. 

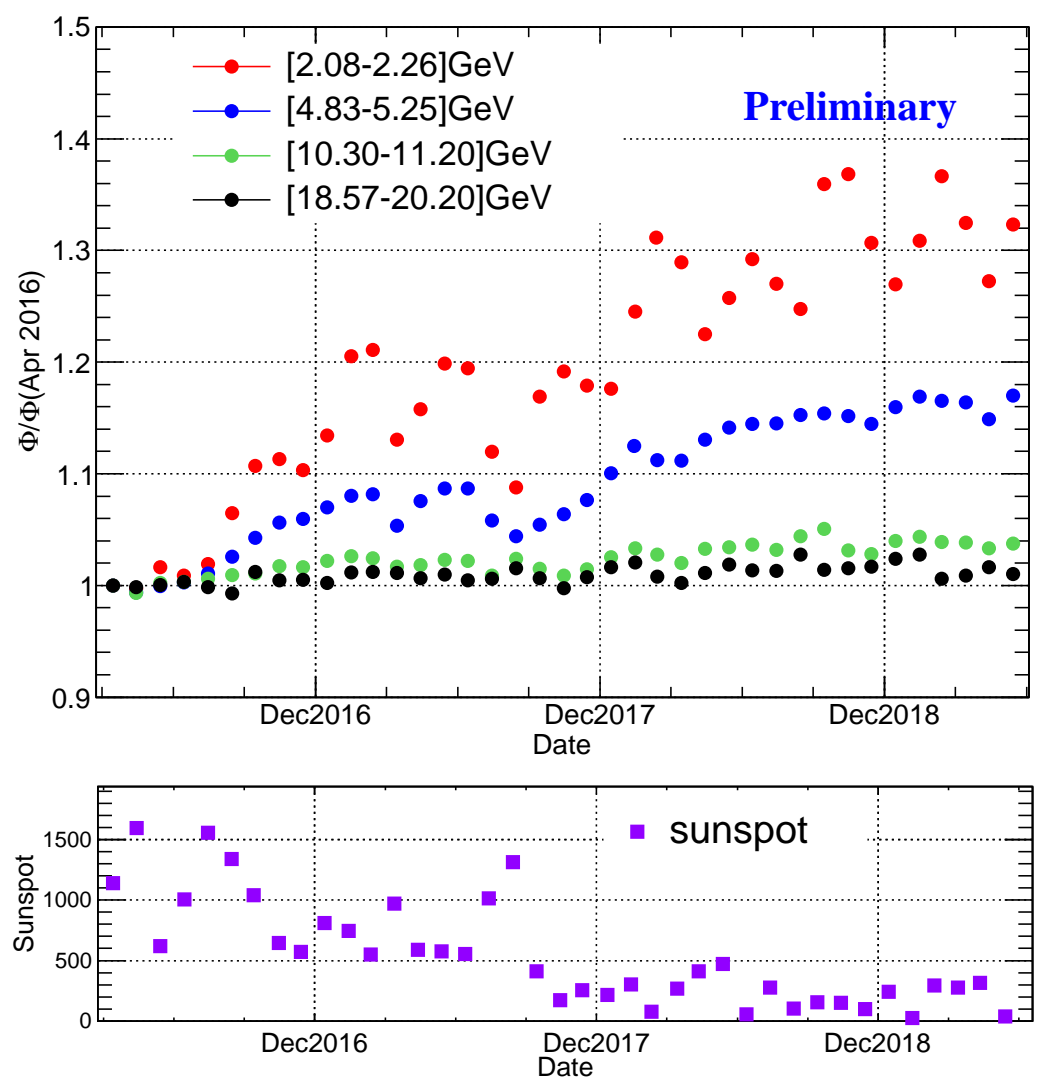

Figure 9: Relative variations of monthly CRE fluxes since April, 2016 [67]. Also shown are the numbers of sunspots which reflect the mean activities of the Sun [68].

\section{Other studies}

The DAMPE data can also be used for studies of problems with broad interests in physics and astrophysics. The nuclear/hadronic interaction cross sections (particularly in specific target material) have relatively large uncertainties, which affect critically the spectral unfolding of the measurements of CR fluxes with the calorimeter detector. Using the on-orbit data we can actually measure the inelastic cross sections of CR nuclei in the BGO detector [71]. Through dividing the BGO calorimeter into two parts, we select proton events which are MIPs in the top part and produce showers in the bottom part. The survival fraction of events in the top part is then related with the inelastic hadronic interaction cross section of protons. Preliminary results show a consistency between the data and the cross sections used in the MC simulation code up to $2 \mathrm{TeV}$ energies [71].

Ultra-heavy nuclei are important indicator of the acceleration sites of CRs [72]. Thanks to the large dynamic range of the PSD of the DAMPE, particles heavier than Ni can be detected and reconstructed. A preliminary analysis using 36 months of the flight data reveals charge peaks of even number nuclei up to $Z=40(\mathrm{Zr})$ [42]. However, the quenching correction of these heavy nuclei needs more careful studies by means of, e.g., ground experiments of ion beams.

Quarks are established as fundamental constituents of hadrons in the standard model of particle 


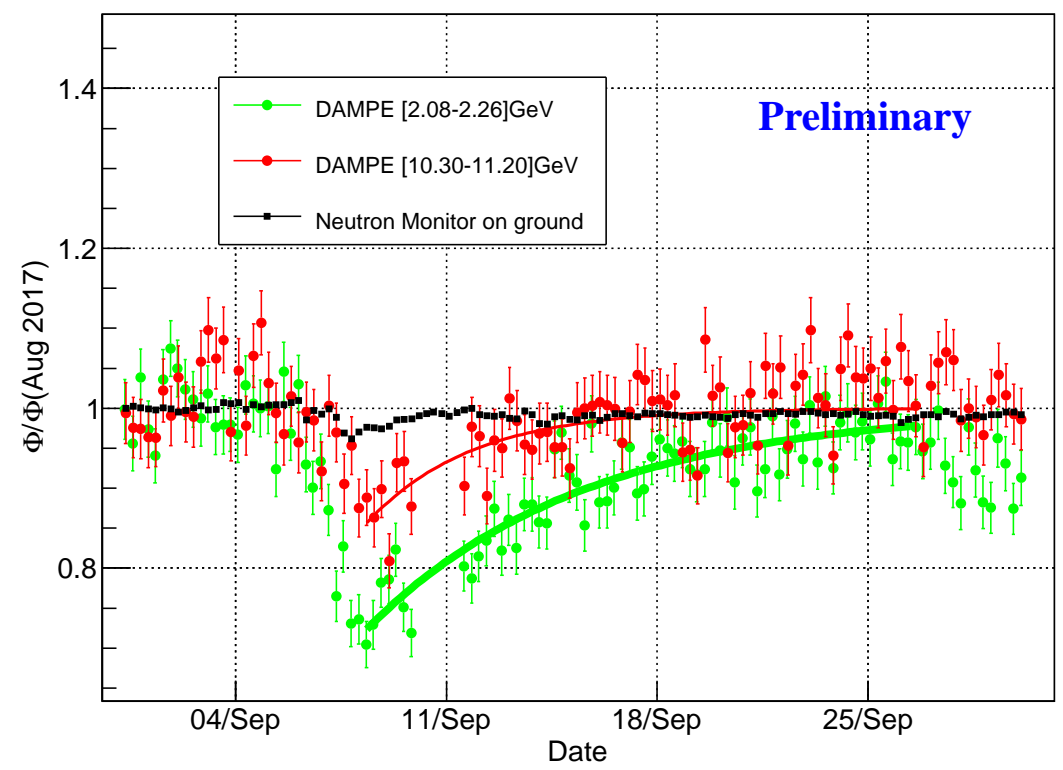

Figure 10: Time profile of relative CRE fluxes in September, 2017 when a giant solar flare occured which resulted in clear Forbush decreases [67].

physics. They have fractional charges of $1 / 3$ or $2 / 3$, but do not appear individually according to the quark confinement theory. Observations of fraction charge particles are important in probing new physics beyond the standard model. We use MIP trigger events to do such searches of fractional charge particles. The analysis method and progress can be found in [42].

\section{Conclusion}

The DAMPE mission has operated in space very smoothly since its launch. The detector works continuously and stably, with over 6 billion CR events being recorded in its 3.5 year run. Careful calibration using the flight data has been made, which verifies the designed performance of the instrument and ensures good measurements of the charge, direction, energy, and type of incident particles. Those physical quantities are the basis of the science analysis.

Precise measurement of the CRE spectrum from $25 \mathrm{GeV}$ to $4.6 \mathrm{TeV}$ has been obtained, revealing a significant break at $E_{\mathrm{br}} \sim 0.9 \mathrm{TeV}$ with spectral index chaning from -3.1 (below $E_{\mathrm{br}}$ ) to -3.9 (above $E_{\mathrm{br}}$ ) [16]. Compared with previous measurements, the DAMPE CRE spectrum improves in better energy resolution, lower background contamination, and wider energy coverage. The CR proton specrum from $40 \mathrm{GeV}$ to $100 \mathrm{TeV}$ is also measured, confirming the spectral hardening at hundreds of $\mathrm{GeV}$ and revealing a spectral softening at $13.6 \mathrm{TeV}$. These results have very interesting implications in modeling of CRs. Analyses of the energy spectra of heavier nuclei, CR anisotropies, the hadronic interaction cross sections, the time evolution of low energy CREs, the line-like $\gamma$-ray emission, as well as exotic strange particles are on-going. With the continuous operation of the mission and accumulation of data, it is expected that the physical results from DAMPE 
can remarkably advance our understanding about the nature of DM particles and the high-energy astrophysical phenomena.

\section{References}

[1] J. Chang, Dark Matter Particle Explorer: The First Chinese Cosmic Ray and Hard Gamma-ray Detector in Space, Chinese Journal of Space Science 34 (2014) 550.

[2] J. Chang et al. [DAMPE Collaboration], The DArk Matter Particle Explorer mission, Astropart. Phys. 95 (2017) 6 [arXiv:1706.08453].

[3] Y. Yu et al., The plastic scintillator detector for DAMPE, Astropart. Phys. 94 (2017) 1 [arXiv:1703.00098].

[4] P. Azzarello et al., The DAMPE silicon-tungsten tracker, Nucl. Instrum. Methods Phys. Res. A 831 (2016) 378.

[5] Z. Zhang et al., Design of a high dynamic range photomultiplier base board for the BGO ECAL of DAMPE, Nucl. Instrum. Methods Phys. Res. A 780 (2015) 21.

[6] M. He et al., GEANT4 Simulation of Neutron Detector for DAMPE, Acta Astron. Sin. 57 (2016) 1.

[7] G. Ambrosi et al. [DAMPE Collaboration], The on-orbit calibration of DArk Matter Particle Explorer, Astropart. Phys. 106 (2019) 18.

[8] Y. L. Zhang et al., The status of DAMPE satellite in space, Proc. Sci. ICRC2019 (2019) 166.

[9] M. Ding et al., Calibration of the DAMPE Plastic Scintillator Detector and its on-orbit performance, Res. Astron. Astrophys. 19 (2019) 047 [arXiv:1810.09901].

[10] P. X. Ma et al., A Method of Alignment of the Plastic Scintillator Detector of DAMPE, Res. Astron. Astrophys. 19 (2019) 082 [arXiv:1808.05720].

[11] T. Dong et al., Charge measurement of cosmic ray nuclei with the plastic scintillator detector of DAMPE, Astropart. Phys. 105 (2019) 31 [arXiv:1810.10784].

[12] A. Tykhonov et al. [DAMPE Collaboration], Internal alignment and position resolution of the silicon tracker of DAMPE determined with orbit data, Nucl. Instrum. Meth. A 893 (2018) 43 [arXiv:1712.02739].

[13] Z. Zhang et al., The calibration and electron energy reconstruction of the BGO ECAL of the DAMPE detector, Nucl. Instrum. Meth. A 836 (2016) 98 [arXiv:1602.07015].

[14] W. Jiang et al., Boresight Alignment with DAMPE, Proc. Sci. ICRC2019 (2019) 567.

[15] J. Chang et al., Resolving electrons from protons in ATIC, Adv. Space Res. 42 (2008) 431.

[16] G. Ambrosi et al. [DAMPE Collaboration], Direct detection of a break in the teraelectronvolt cosmic-ray spectrum of electrons and positrons, Nature 552 (2017) 63 [arXiv:1711.10981].

[17] D. Droz et al., Neural Networks for Electron Identification with DAMPE, Proc. Sci. ICRC2019 (2019) 064.

[18] M. Aguilar et al. [AMS Collaboration], Precision Measurement of the $\left(e^{+}+e\right)$ Flux in Primary Cosmic Rays from $0.5 \mathrm{GeV}$ to $1 \mathrm{TeV}$ with the Alpha Magnetic Spectrometer on the International Space Station, Phys. Rev. Lett. 113 (2014) 221102.

[19] S. Abdollahi et al. [Fermi-LAT Collaboration], Cosmic-ray electron-positron spectrum from $7 \mathrm{GeV}$ to 2 TeV with the Fermi Large Area Telescope, Phys. Rev. D 95 (2017) 082007 [arXiv:1704.07195]. 
[20] O. Adriani et al. [CALET Collaboration], Energy Spectrum of Cosmic-Ray Electron and Positron from $10 \mathrm{GeV}$ to $3 \mathrm{TeV}$ Observed with the Calorimetric Electron Telescope on the International Space Station, Phys. Rev. Lett. 119 (2017) 181101 [arXiv:1712.01711].

[21] F. Aharonian et al. [H.E.S.S. Collaboration], The energy spectrum of cosmic-ray electrons at TeV energies, Phys. Rev. Lett. 101 (2008) 261104 [arXiv:0811.3894].

[22] F. Aharonian et al. [H.E.S.S. Collaboration], Probing the ATIC peak in the cosmic-ray electron spectrum with H.E.S.S, Astron. Astrophys. 508 (2009) 561 [arXiv:0905.0105].

[23] Q. Yuan et al., Interpretations of the DAMPE electron data, arXiv:1711.10989.

[24] Q. Yuan and L. Feng, Dark Matter Particle Explorer observations of high-energy cosmic ray electrons plus positrons and their physical implications, Sci. China Phys. Mech. Astron. 61 (2018) 101002 [arXiv:1807.11638].

[25] K. Fang, X. J. Bi and P. F. Yin, Explanation of the knee-like feature in the DAMPE cosmic $e^{-}+e^{+}$ energy spectrum, Astrophys. J. 854 (2018) 57 [arXiv:1711.10996].

[26] O. Adriani et al. [PAMELA Collaboration], PAMELA Measurements of Cosmic-ray Proton and Helium Spectra, Science 332 (2011) 69 [arXiv:1103.4055].

[27] M. Aguilar et al. [AMS Collaboration], Precision Measurement of the Proton Flux in Primary Cosmic Rays from Rigidity 1 GV to 1.8 TV with the Alpha Magnetic Spectrometer on the International Space Station, Phys. Rev. Lett. 114 (2015) 171103.

[28] M. Aguilar et al. [AMS Collaboration], Precision Measurement of the Helium Flux in Primary Cosmic Rays of Rigidities 1.9 GV to 3 TV with the Alpha Magnetic Spectrometer on the International Space Station, Phys. Rev. Lett. 115, no. 21, 211101 (2015).

[29] A. D. Panov et al., Energy Spectra of Abundant Nuclei of Primary Cosmic Rays from the Data of ATIC-2 Experiment: Final Results, Bull. Russ. Acad. Sci. Phys. 73 (2009) 564 [arXiv:1101.3246].

[30] H. S. Ahn et al., Discrepant hardening observed in cosmic-ray elemental spectra, Astrophys. J. 714 (2010) L89 [arXiv:1004.1123].

[31] Y. S. Yoon et al., Proton and Helium Spectra from the CREAM-III Flight, Astrophys. J. 839 (2017) 5 [arXiv:1704.02512].

[32] E. Atkin et al., New Universal Cosmic-Ray Knee near a Magnetic Rigidity of 10 TV with the NUCLEON Space Observatory, JETP Lett. 108 (2018) 5 [arXiv:1805.07119].

[33] O. Adriani et al. [CALET Collaboration], Direct Measurement of the Cosmic-Ray Proton Spectrum from $50 \mathrm{GeV}$ to $10 \mathrm{TeV}$ with the Calorimetric Electron Telescope on the International Space Station, Phys. Rev. Lett. 122 (2019) 181102 [arXiv:1905.04229].

[34] C. Yue et al., Measurement of cosmic ray proton spectrum with the Dark Matter Particle Explorer, Proc. Sci. ICRC2019 (2019) 163.

[35] G. D’Agostini, A Multidimensional unfolding method based on Bayes' theorem, Nucl. Instrum. Meth. A 362 (1995) 487.

[36] V. I. Zatsepin and N. V. Sokolskaya, Three component model of cosmic ray spectra from 100-gev up to 100-pev, Astron. Astrophys. 458 (2006) 1 [astro-ph/0601475].

[37] W. Liu, Y. Q. Guo and Q. Yuan, Indication of nearby source signatures of cosmic rays from energy spectra and anisotropies, arXiv:1812.09673 (2018). 
[38] M. Di Santo et al., Helium spectrum in the cosmic rays measured by the DAMPE detector, Proc. Sci. ICRC2019 (2019) 058.

[39] M. Aguilar et al. [AMS Collaboration], Observation of the Identical Rigidity Dependence of He, C, and $O$ Cosmic Rays at High Rigidities by the Alpha Magnetic Spectrometer on the International Space Station, Phys. Rev. Lett. 119 (2017) 251101.

[40] O. Adriani et al., Measurements of cosmic-ray proton and helium spectra with the PAMELA calorimeter, Adv. Space Res. 51 (2013) 219.

[41] I. DeMitri et al., Measurement of the cosmic-ray proton + helium spectrum with DAMPE, Proc. Sci. ICRC2019 (2019) 148.

[42] Y. P. Zhang et al., Elemental analysis of Cosmic-Ray flux with DAMPE, Proc. Sci. ICRC2019 (2019) 165.

[43] L. B. Wu et al., The Selection and energy validation of heavy ions based on DAMPE orbit data, Proc. Sci. ICRC2019 (2019) 156.

[44] A. Tykhonov et al., TeV-PeV hadronic simulations with DAMPE, Proc. Sci. ICRC2019 (2019) 143.

[45] M. Amenomori [Tibet AS-gamma Collaboration], Anisotropy and Corotation of Galactic Cosmic Rays, Science 314 (2006) 439 [astro-ph/0610671].

[46] O. Adriani et al., Search for Anisotropies in Cosmic-ray Positrons Detected by the PAMELA Experiment, Astrophys. J. 811 (2015) 21 [arXiv:1509.06249].

[47] S. Abdollahi et al. [Fermi-LAT Collaboration], Search for Cosmic-Ray Electron and Positron Anisotropies with Seven Years of Fermi Large Area Telescope Data, Phys. Rev. Lett. 118 (2017) 091103 [arXiv:1703.01073].

[48] M. Ajello et al. [Fermi-LAT Collaboration], A Search for Cosmic-ray Proton Anisotropy with the Fermi Large Area Telescope, arXiv:1903.02905.

[49] G. La Vacca [AMS-02 Collaboration], Search for Cosmic Ray Anisotropy with the Alpha Magnetic Spectrometer on the International Space Station, arXiv:1612.08957.

[50] O. Adriani et al. [PAMELA Collaboration], Ten years of PAMELA in space, Riv. Nuovo Cim. 40 (2017) 1 [arXiv:1801.10310].

[51] M. Munoz et al., Anisotropy searches with DAMPE, Proc. Sci. ICRC2019 (2019) 113.

[52] X. Li et al., Recent Gamma-ray Results from DAMPE, Proc. Sci. ICRC2019 (2019) 576.

[53] B. P. Abbott et al. [LIGO Scientific and Virgo Collaborations], Observation of Gravitational Waves from a Binary Black Hole Merger, Phys. Rev. Lett. 116 (2016) 061102 [arXiv:1602.03837].

[54] M. G. Aartsen et al. Multimessenger observations of a flaring blazar coincident with high-energy neutrino IceCube-170922A, Science 361 (2018) eaat1378 [arXiv:1807.08816].

[55] Z. L. Xu et al., An algorithm to resolve $\gamma$-rays from charged cosmic rays with DAMPE, Res. Astron. Astrophys. 18 (2018) 027 [arXiv:1712.02939].

[56] Z. Q. Shen et al., Search for a gamma-ray line feature with DAMPE, Proc. Sci. ICRC2019 (2019) 601.

[57] K. K. Duan et al., DmpIRFs and DmpST: DAMPE Instrument Response Functions and Science Tools for Gamma-Ray Data Analysis, arXiv:1904.13098. 
[58] M. Ackermann et al. [Fermi-LAT Collaboration], Search for Gamma-ray Spectral Lines with the Fermi Large Area Telescope and Dark Matter Implications, Phys. Rev. D 88 (2013) 082002 [arXiv:1305.5597].

[59] M. Ackermann et al. [Fermi-LAT Collaboration], Updated search for spectral lines from Galactic dark matter interactions with pass 8 data from the Fermi Large Area Telescope, Phys. Rev. D 91 (2015) 122002 [arXiv:1506.00013].

[60] A. A. Abdo et al. [Fermi-LAT Collaboration], The Second Fermi Large Area Telescope Catalog of Gamma-ray Pulsars, Astrophys. J. Suppl. 208 (2013) 17 [arXiv:1305.4385].

[61] M. Munoz et al., Gamma-ray pulsars with DAMPE, Proc. Sci. ICRC2019 (2019) 587.

[62] M. Aguilar et al. [AMS Collaboration], Observation of Fine Time Structures in the Cosmic Proton and Helium Fluxes with the Alpha Magnetic Spectrometer on the International Space Station, Phys. Rev. Lett. 121 (2018) 051101.

[63] M. Aguilar et al. [AMS Collaboration], Observation of Complex Time Structures in the Cosmic-Ray Electron and Positron Fluxes with the Alpha Magnetic Spectrometer on the International Space Station, Phys. Rev. Lett. 121 (2018) 051102.

[64] R. Munini et al., Evidence of Energy and Charge Sign Dependence of the Recovery Time for the 2006 December Forbush Event Measured by the PAMELA Experiment, Astrophys. J. 853 (2018) 76 [arXiv:1803.06166].

[65] M. Martucci et al., Proton Fluxes Measured by the PAMELA Experiment from the Minimum to the Maximum Solar Activity for Solar Cycle 24, Astrophys. J. 854 (2018) L2 [arXiv:1801.07112].

[66] A. Bruno et al. [PAMELA Collaboration], Solar energetic particle events observed by the PAMELA mission, Astrophys. J. 862 (2018) 97 [arXiv:1807.10183].

[67] J. J. Zang et al., Observation of time evolution of cosmic ray electron and positron fluxes with DAMPE, Proc. Sci. ICRC2019, this conference (2019).

[68] http://www.sidc.be/silso/home

[69] C. R. Zhu, Q. Yuan and D. M. Wei, Studies on cosmic ray nuclei with Voyager, ACE and AMS-02: I. local interstellar spectra and solar modulation, Astrophys. J. 863 (2018) 119 [arXiv:1807.09470].

[70] L. L. Zhao and H. Zhang, Transient Galactic Cosmic-ray Modulation during Solar Cycle 24: A Comparative Study of Two Prominent Forbush Decrease Events, Astrophys. J. 827 (2016) 13.

[71] Y. F. Wei et al., Hadronic cross section validation in the DAMPE experiment, Proc. Sci. ICRC2019 (2019) 149.

[72] W. R. Binns et al., Abundances of ultraheavy elements in the cosmic radiation - results from HEAO 3 , Astrophys. J. 346 (1989) 997. 